\title{
Guidelines
}

European

Thyroid Journal
Eur Thyroid J 2013;2:76-82

DOI: $10.1159 / 000351007$
Received: February 6, 2013

Accepted after revision: March 18, 2013

Published online: May 7, 2013

\section{European Thyroid Association Guidelines for the Diagnosis and Treatment of Thyrotropin-Secreting Pituitary Tumors}

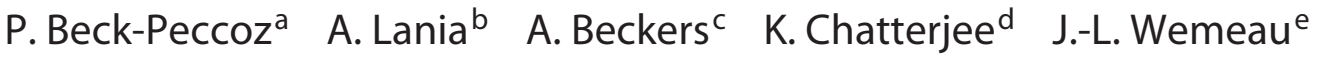

\begin{abstract}
a Department of Clinical Sciences and Community Health, University of Milan, Endocinology and Diabetology Unit, Fondazione IRCCS Cà Granda Policlinico and b Dipartimento di Biotecnologie Mediche e Medicina Traslazionale, University of Milan, Endocrine Unit, Istituto Clinico Humanitas IRCCS, Rozzano, Milan, Italy; 'Service d'Endocrinologie, Centre Hospitalier Universitaire de Liège, Université de Liège, Liège, Belgium; ${ }^{d}$ Institute of Metabolic Science, Metabolic Research Laboratories, Addenbrooke's Hospital, University of Cambridge, Cambridge, UK; ${ }^{e} \mathrm{CHRU}$, Hôpital Claude-Huriez, Clinique Endocrinologique Marc-Linquette, Lille, France
\end{abstract}

\section{Key Words}

Thyrotropin-secreting pituitary adenoma $\cdot$ Central hyperthyroidism - Thyrotropin - Resistance to thyroid hormones · Transsphenoidal surgery · Somatostatin analogs

\begin{abstract}
Hyperthyroidism is mainly due to autoimmune thyroid disorders or toxic goiter, and very rarely to the presence of thyrotropin (TSH)-secreting pituitary adenomas (TSHomas). These tumors are characterized by high levels of circulating free thyroid hormones (FT4 and FT3) in the presence of nonsuppressed serum TSH concentrations. Failure to correctly diagnose TSHomas may result in inappropriate thyroid ablation, which results in a significant increase of pituitary tumor mass. The diagnosis is mainly achieved by measuring TSH after T3 suppression and TRH stimulation tests. These dynamic tests, together with pituitary imaging and genetic testing are useful in distinguishing TSHomas from the syndromes of resistance to thyroid hormone action. The treatment of choice is surgery. In cases of surgical failure, somatostatin analogs have been found to be effective in normalizing TSH secretion in more than $90 \%$ of patients.
\end{abstract}

Copyright $\odot 2013$ European Thyroid Association Published by S. Karger AG, Basel

\section{Introduction}

The syndrome of 'inappropriate secretion of TSH' was the term coined originally [1] to indicate two forms of central hyperthyroidism, i.e. thyrotropin (TSH)-secreting pituitary adenomas (TSHomas) and resistance to thyroid hormone action (RTH) [2-5]. Both forms are characterized by high levels of FT4 and FT3 in the presence of measurable TSH concentrations, a biochemical picture which is in contrast to primary hyperthyroidism (Graves' disease, toxic goiter, thyrotoxicosis factitia and others) where TSH levels are always undetectable. Patients with TSHoma are clinically hyperthyroid, while RTH patients are generally euthyroid (so-called generalized RTH). However, in a minority of RTH patients, thyrotoxic features such as weight loss, tremor, tachycardia and palpitations, anxiety, insomnia and heat intolerance in adults have been described with such individuals being deemed to have predominant central or pituitary resistance [5].

The first case of central hyperthyroidism due to a TSHoma was reported in 1960 [6]. Since then, the availability of ultrasensitive immunometric TSH assays, which are routinely performed in association with direct measurement of circulating FT4 and FT3, has led to increasing recognition of patients with normal or elevated TSH levels in the presence of high free thyroid hormone levels.

\begin{tabular}{ll}
\hline KARGER & @ 2013 European Thyroid Association \\
& Published by S. Karger AG, Basel \\
& $2235-0640 / 13 / 0022-0076 \$ 38.00 / 0$ \\
$\begin{array}{l}\text { E-Mail karger@karger.com } \\
\text { www.karger.com/etj }\end{array}$ &
\end{tabular}

Paolo Beck-Peccoz, MD

Pad. Granelli

Via F. Sforza 35

IT-20122 Milano (Italy)

E-Mail paolo.beckpeccoz@unimi.it 
Central hyperthyroidism represents a diagnostic and therapeutic challenge. In fact, failure to correctly diagnose these different disorders may result in significant adverse consequences, such as inappropriate thyroid ablation in patients with TSHomas or unnecessary pituitary surgery in patients with RTH.

\section{Methods}

The taskforce for this guideline was instituted by the executive committee of the European Thyroid Association. References were searched in PubMed using the following search terms: TSH-secreting pituitary adenoma, central hyperthyroidism, TSH, syndromes of resistance to thyroid hormones, transsphenoidal surgery, somatostatin analogs. The Grading of Recommendations Assessment, Development and Evaluation system was used to grade the quality of evidence and the strength of recommendations $[7,8]$. It consists of 2 scorings, namely quality of evidence (low $=+$; moderate $=++$; high $=+++$ ) and strength of recommendation (strong; weak). The draft guideline was posted on the European Thyroid Association website for 1 month for its members to comment. The comments were discussed in the guideline taskforce and the guideline modified accordingly before final approval by the guideline committee and the European Thyroid Association executive committee.

\section{Background}

\section{Epidemiology}

Since 1960 more than 450 cases of TSHoma have been published, the prevalence of these adenomas being around one case per million. TSHomas account for about $0.5-3 \%$ of all pituitary adenomas [3,9], a figure probably underestimated owing to recently published studies on the epidemiology of pituitary tumors [10-12]. Moreover, an increase in the number of TSHomas diagnosed in the last 3 decades has been described, a finding probably due to both practitioner awareness and the introduction of ultrasensitive immunometric TSH assays that are able to clearly distinguish between suppressed and normal circulating TSH levels. Most patients are diagnosed around the fifth-sixth decade of life with no gender difference [3]. Familial cases of TSHoma have been reported as part of the multiple endocrine neoplasia type 1 syndrome [13], but also in one familial isolated pituitary adenoma family with AIP mutations [14].

\section{Pathogenesis}

The molecular mechanisms involved in thyrotrope adenomatous transformation are still unknown. It has been demonstrated that TSHomas are monoclonal in origin [15], thus suggesting that the development of TSHomas might be explained by the presence of a transforming event providing gain of proliferative function followed by secondary mutations or alterations favoring tumor progression [16]. However, to date, no mutations either in oncogenes commonly activated in human cancer (e.g. Ras) or in antioncogenes, such as p53 and menin, have been identified $[17,18]$. Moreover, no somatic TRH receptor or $G$ protein subunit gene mutations have been reported [18]. Thyrotrope tumor refractoriness to the inhibitory action of thyroid hormones suggested the possible involvement of somatic thyroid hormone receptor (TR) defects or mutations in thyrotrope transformation, prompting a search for alterations in TR function. However, absence of TR $1, T R \alpha 2$ and TR $\beta 1$ expression was reported only in one TSHoma [19]. Recently, aberrant alternative splicing of TR $\beta 2$ mRNA, generating a TR $\beta$ variant lacking T3 binding activity, as well as the aberrant expression of TR $\beta 4$ isoform have been proposed as mechanisms for impaired T3-dependent negative regulation of TSH secretion in tumoral tissue $[20,21]$.

\section{Pathology}

TSHomas are usually benign tumors, supported by the fact that, to date, transformation of a TSHoma into a carcinoma with multiple metastases has only been described in a few patients [22]. These adenomas are often large and invasive lesions and very fibrous; larger and more invasive TSHomas are more frequently observed in patients who have undergone thyroid ablation [3]. Up to $70-80 \%$ of TSHomas secrete only TSH, particularly the microadenomas [23], though hormone production is often accompanied by an unbalanced hypersecretion of the glycoprotein hormone $\alpha$-subunit ( $\alpha$-GSU) [3]. Interestingly, $20-25 \%$ of TSHomas are mixed adenomas, characterized by concomitant hypersecretion of other anterior pituitary hormones, mainly growth hormone or prolactin (PRL) [3]. TSH-secreting adenomatous cells are usually chromophobe in appearance. Ultrastructurally, the well-differentiated adenomatous thyrotropes resemble the normal cell type. Conversely, poorly differentiated adenomas are composed of elongated cells with irregular nuclei, poorly developed RER, and sparse small secretory granules usually located along the cell membrane [2]. Finally, there is clear in vitro evidence that almost all TSHomas express a variable number of somatostatin receptors [24]. These findings prompted the use of somatostatin analogs in TSHoma treatment. 


\section{Clinical Manifestations}

Patients with TSHoma are characterized by increased circulating levels of both free T4 and T3, therefore associated with signs and symptoms of hyperthyroidism. Most of these patients have a long history of hyperthyroidism, which is frequently misdiagnosed as Graves' disease, and may have been inappropriately treated by thyroidectomy or radioiodine therapy $[2,3]$.

Clinical features of hyperthyroidism are sometimes inappropriately milder than expected given the circulating level of thyroid hormones. In the case of mixed TSH/ growth hormone tumors, the signs and symptoms of acromegaly may mask those of hyperthyroidism [3]. Contrary to what is observed in patients with primary hyperthyroidism, the deleterious effects of thyroid hormone excess on the heart (i.e. atrial fibrillation and/or cardiac failure) are less frequently observed. Conversely, uni- or multinodular goiter is described in the majority of patients and recurrence of goiter is frequent after partial thyroidectomy $[3,25,26]$. Interestingly, progression of cases to frank toxic nodular goiter is very infrequent [2]. The presence of differentiated thyroid cancer is reported in very few cases $[3,22]$. Signs of orbitopathy or pretibial myxedema are absent, though exophthalmos due to orbital invasion by the pituitary tumor or TSHoma arising in patients who subsequently developed autoimmune thyroiditis has been described.

Signs and symptoms of hypopituitarism should be carefully evaluated. The gonadal axis is frequently affected. Menstrual disorders are present in all females with mixed TSH/PRL tumors and in one third of those with pure TSHoma. Central hypogonadism, delayed puberty and decreased libido have been described in male patients with either 'pure' or mixed TSH/PRL adenomas.

Since the majority of TSHomas are large and invasive adenomas, signs and symptoms of expanding tumor mass (i.e. visual field defects, loss of vision, headache, partial or total hypopituitarism) are predominant in many patients [27].

\section{Recommendations}

\section{Diagnostic Principles}

When Should TSHoma Be Suspected?

(1) High concentrations of circulating total or free thyroid hormones in the presence of non-suppressed TSH levels characterize TSHomas. In interpreting these findings, the possible presence of factors, which potentially interfere with the measurement methods of either thyroid hormones or TSH, must be ruled out. In fact, the presence of anti-iodothyronine auto-antibodies (anti-T4 and/or anti-T3) or abnormal forms of albumin or transthyretin may cause T4 and/or T3 to be falsely elevated, particularly when 'one-step' analog methods are employed [2, 28, 29]. Moreover, spuriously increased levels of TSH might be due to circulating heterophilic antibodies, i.e. antibodies directed against mouse gamma-globulins employed in TSH immunometric assay [3]. Finally, patients that have undergone thyroid ablation 'by mistake' may present as patients with unreasonable high TSH that thyroxine replacement cannot restore maintaining normal FT4.

Quality of evidence: high (+++); strength of recommendations: strong.

(2) Signs and symptoms of hyperthyroidism are usually mild and frequently masked by those of concomitant hypersecretion of other pituitary hormones (mixed adenomas) $[23,25,29]$.

Quality of evidence: moderate $(++)$; strength of recommendations: weak.

How Is the Diagnosis of TSHoma Established?

(1) Although the circulating levels of serum TSH and thyroid hormones are extremely variable [23, 25, 26, 29, 30] the finding of measurable TSH levels in the presence of high FT4 and FT3 concentrations is the hallmark of TSHomas.

Quality of evidence: high $(+++)$; strength of recommendations: strong.

(2) MRI of the hypothalamic-pituitary region allows the discovery of macro-adenomas (about $80 \%$ of patients) and microadenomas in the anterior pituitary. However, ectopic tumors in the pharyngeal region have been reported [30]. Cases with the clinical and biochemical characteristics of TSHoma but negative MR imaging, presumably reflecting microadenomas that cannot be visualized, have been described.

Quality of evidence: moderate $(++)$; strength of recommendations: weak.

(3) Both stimulatory and inhibitory tests should be employed in the differential diagnosis of TSHomas [21, $23,24]$. Blunted TSH responses to TRH stimulation (200 $\mu \mathrm{g}$ bolus intravenously, sampling at $0,20,60,90$ and $120 \mathrm{~min}$ ) were seen in $90 \%$ of patients with TSHomas, whilst complete inhibition of TSH following administration of T3 suppression test $(80-100 \mu \mathrm{g} /$ day divided in 3 administrations for 10 days, sampling at 0,5 and 10 days) was never reported in these patients [30].

Quality of evidence: high $(+++)$; strength of recommendations: strong.
Beck-Peccoz/Lania/Beckers/Chatterjee/ Wemeau 
(4) The measurement of serum glycoprotein hormone alpha-subunit ( $a-G S U$ ) shows high circulating levels in about $70 \%$ of patients with TSHoma, in particular in those with a macroadenoma [23]. A high $\alpha$-GSU/TSH molar ratio $\{[\alpha-G S U(\mu \mathrm{g} / \mathrm{l}) / \mathrm{TSH}(\mathrm{mU} / \mathrm{l})] \times 10\}$ is present in about $80 \%$ of TSHomas. However, calculation of the molar ratio cutoff which suggests TSHoma must take the circulating levels of other pituitary glycoprotein hormones (TSH, luteinizing hormone and follicle-stimulating hormone) into account. Thus, molar ratios ranging from 0.3 in normal men to 29.1 in postmenopausal women have been described [31]. Therefore, it is no longer tenable to use a single molar ratio cutoff of $>1.0$ as indicative of TSHoma.

Quality of evidence: moderate (++); strength of recommendations: weak.

(5) A number of parameters of thyroid hormone action may help in determining the degree of peripheral tissue hyperthyroidism. In particular, bone (carboxy-terminal cross-linked telopeptide of type I collagen) and liver (sex hormone-binding globulin) parameters appear to be useful in differentiating TSHoma patients from those with RTH [32,33]. Other biomarkers, such as cholesterol, LDL, triglycerides, ferritin, erythrocyte microcytosis, resting energy expenditure and cardiac function parameters are of limited help in the diagnosis of TSHomas.

Quality of evidence: low (+); strength of recommendations: weak.

How to Perform the Differential Diagnosis with RTH?

Several diagnostic steps should be carried out to differentiate the two forms of central hyperthyroidism, i.e. to distinguish a TSHoma from RTH $[2,4,5,30]$ (fig. 1).

(1) The possible presence of neurological signs and symptoms (visual defects, headache) or clinical features (acromegaly, galactorrhea, amenorrhea) of concomitant hypersecretion of other pituitary hormones points to the presence of a TSHoma. The presence of pituitary lesions on MRI strongly supports the diagnosis of TSHoma. However, the usefulness of such imaging is limited by the known background prevalence of pituitary abnormalities (e.g. empty sella, pituitary incidentalomas) in healthy subjects.

Quality of evidence: moderate $(++)$; strength of recommendations: weak.

(2) A partial inhibition of TSH secretion after T3 supression test is seen only in RTH patients $[5,29]$. The TSH response to TRH stimulation is usually preserved in RTH patients [5].

Quality of evidence: moderate $(++)$; strength of recommendations: weak.

Diagnosis and Treatment of TSHomas
(3) Since RTH occurs as a dominantly-inherited disorder in $\sim 70 \%$ of cases, the finding of a similar thyroid biochemical phenotype in other first-degree relatives is highly suggestive of RTH. Mutations in the thyroid hormone receptor beta gene are identified in $\sim 75-80 \%$ of RTH, especially familial cases.

Quality of evidence: moderate (++); strength of recommendations: weak.

(4) No significant differences in age, sex, previous thyroid ablation, TSH levels, or thyroid hormone concentrations occur between patients with TSHoma and those with RTH. Serum TSH levels within the normal range are more frequently found in RTH, while high a-GSU concentrations and/or high $\alpha$-GSU/TSH molar ratios are typically present in patients with TSH-secreting tumors. Circulating sex hormone-binding globulin levels are usually in the thyrotoxic range in patients with TSH-secreting tumors (unless concomitant $\mathrm{GH}$ hypersecretion is present), whilst they are normal in $\mathrm{RTH}$, even in patients with predominant central or pituitary resistance [32].

Quality of evidence: moderate $(++)$; strength of recommendations: weak.

(5) Chronic administration of long-acting somatostatin analogs (20-30 mg i.m. every 28 days for 2-4 months, sampling at 0 and every 28 days just before the new injection) in patients with central hyperthyroidism caused a marked decrease of free T4 and free T3 levels in all but one patient with TSHoma, while patients with RTH did not respond at all [34]. Thus, administration of long-acting somatostatin analogs for at least 2 months can be useful in the differential diagnosis in problematic cases of central hyperthyroidism. Furthermore, these observations also indicate that chronic administration of somatostatin analogs to control thyrotoxic symptoms in RTH patients, is not beneficial [34].

Quality of evidence: low (+); strength of recommendations: weak.

\section{Therapeutic Principles}

What Is the Optimal Treatment for TSHomas?

(1) Surgical removal of the adenoma is the first-line therapy for TSHomas, with transsphenoidal or subfrontal adenomectomy being able to completely remove the tumor and to restore normal pituitary/thyroid function. Complete removal of the tumor is achieved in the majority of patients with microadenoma, whereas no more than $60 \%$ of patients with macroadenoma may be cured. The reasons of surgical failure are marked fibrosis within the adenoma and its frequent extra- and parasellar extension $[23,25,30,35]$. 


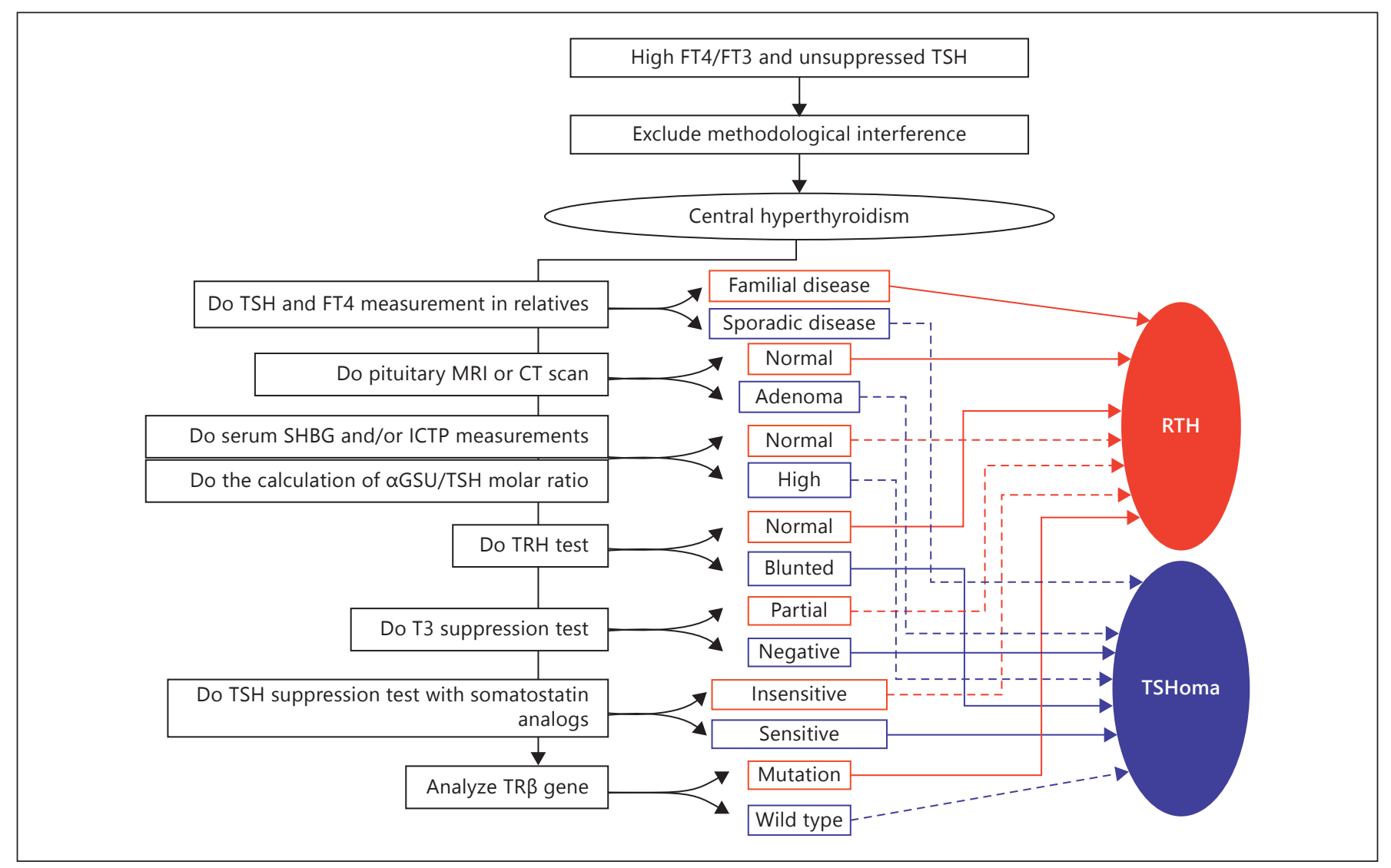

Fig. 1. Flow-chart for the differential diagnosis between TSHoma and $\mathrm{RTH}$. After exclusion of methodological interferences, central hyperthyroidism is confirmed. A panel of clinical, biochemical and genet- ic tests may be necessary to reach a differential diagnosis. Red findings are more consistent with RTH and blue findings with TSHoma. Solid lines indicate findings giving a stronger diagnostic indication.
Quality of evidence: high (+++); strength of recommendations: strong.

(2) If surgery and medical treatment are contraindicated or declined, pituitary fractionated stereotaxic radiotherapy or radiosurgery might be considered. However, no data on the success rate of radiotherapy are available apart from a successful experience of an invasive TSHoma treated by two-stage operation and gamma knife [24].

Quality of evidence: low (+); strength of recommendations: weak.

(3) Medical treatment of TSHomas is mainly based on the administration of somatostatin analogs that have been demonstrated to be highly effective in reducing TSH secretion from neoplastic thyrotropes. Treatment with long-acting somatostatin analogs, such as octreotide LAR $^{\circledR}$ or lanreotide $S R^{\circledR}$ or lanreotide Autogel ${ }^{\circledR}$, induces a reduction in TSH and $\alpha$-GSU secretion in almost all cases, with restoration of the euthyroid state in the majority of them $[23,25,29,30]$. Circulating thyroid hormone lev- els normalized in more than $90 \%$ of patients and goiter size is significantly reduced by somatostatin analog therapy in about $30 \%$ of cases $[23,24,30]$. Somatostatin analog treatment induces a significant tumor mass shrinkage in about $40 \%$ of patients and vision improvement in about $70 \%$ of them $[23,30]$. Cure of one TSHoma by medical treatment with somatostatin analogs has recently been reported [36]. Resistance to somatostatin analog treatment, true escape of TSH secretion from the inhibitory effects of the drugs or discontinuation of treatment due to side effects was documented in a minority of cases. Patients on somatostatin analogs have to be carefully monitored, as untoward side effects, such as cholelithiasis and hyperglycemia, may become manifest. The administered dose should be tailored for each patient, depending on therapeutic response and tolerance including gastrointestinal side effects. The tolerance is usually very good, as gastrointestinal side effects are transient with long-acting analogs. The marked somatostatin-induced suppression of 
TSH secretion and consequent biochemical hypothyroidism seen in some patients may require L-T4 substitution.

Quality of evidence: high (+++); strength of recommendations: strong.

(4) Dopamine type 2 receptors are present in most TSHomas and thus dopamine agonists, such as bromocriptine or cabergoline, have been used in the treatment of affected patients. However, the results are mixed and only partial TSH suppression is seen in the majority of cases.

Quality of evidence: low (+); strength of recommendations: weak.

How to Prepare the Patient for Neurosurgery?

Antithyroid drugs (methimazole or propylthiouracil) or somatostatin analogs, such as octreotide and lanreotide, along with propranolol, should be administered in order to restore euthyroidism before surgery $[23,26,30]$.

Quality of evidence: moderate $(++)$; strength of recommendations: weak.

\section{Which Are the Criteria of Cure of a TSHoma?}

(1) The criteria of cure of patients operated and/or irradiated for TSHoma have not been clearly established. Nevertheless, clinical remission of hyperthyroidism, disappearance of neurological symptoms, resolution of neuroradiological alterations and normalization of thyroid hormones, TSH, or $\alpha$-GSU/TSH molar ratio are taken into account for the evaluation of the efficacy of surgery or radiotherapy. It is obvious that previous thyroid ablation makes some of these criteria not applicable [29]. The restoration of clinical and biochemical euthyroidism in untreated hyperthyroid patients is not per se synonymous of complete removal of tumoural cells, since transient clinical remission accompanied by normalization of thyroid function tests has been observed [35]. Disappearance of neurological signs and symptoms is a good prognostic outcome, but it may occur even after an incomplete debulking of the tumor. Undetectable TSH levels 1 week after surgery are likely to indicate complete adenomectomy, provided that presurgical treatment with antithyroid drugs or daily somatostatin analog injections were stopped at least 10 days before surgery [35]. The most sensitive and specific test to document the complete removal of the adenoma remains the T3 suppression test. In fact, only patients in whom T3 administration completely inhibits basal and TRH-stimulated TSH secretion, appear to be truly cured [35].

Quality of evidence: low (+); strength of recommendations: weak.

\section{How to Perform the Follow-Up of TSHomas?}

(1) No data on recurrence rates of TSHomas in patients judged cured after surgery or radiotherapy have been reported. However, the recurrence of the adenoma does not appear to be frequent, at least in the first years after successful surgery $[23,25,35]$. In general, the patient should be evaluated clinically and biochemically 2 or 3 times the first year postoperatively, and then every year. The evaluation should include the measurement of TSH and circulating free thyroid hormones, as well as that of other pituitary hormones if indicated. Pituitary imaging should be performed every two or three years, but should be promptly done whenever an increase in TSH and thyroid hormone levels, or clinical symptoms occur. In the case of persistent macroadenoma, a close follow-up of visual fields is require to ensure that visual function is not threatened.

Quality of evidence: low (+); strength of recommendations: weak.

\section{Disclosure Statement}

The authors have nothing to disclose.

\section{References}

1 Gershengorn MC, Weintraub BD: Thyrotropin-induced hyperthyroidism caused by selective pituitary resistance to thyroid hormone. A new syndrome of 'inappropriate secretion of TSH'. J Clin Invest 1975;56:633-642.

$\longrightarrow 2$ Beck-Peccoz P, Brucker-Davis F, Persani L, et al: Thyrotropin-secreting pituitary tumors. Endocr Rev 1996;17:610.

-3 Beck-Peccoz P, Persani L, Mannavola D, Campi I: Pituitary tumours: TSH-secreting adenomas. Best Pract Res Clin Endocrinol Metab 2009;23:597-606.
4 Refetoff S, Dumitrescu AM: 2010 Resistance to thyroid hormone; in: Thyroid Disease Manager, chapter 16D (http://www.thyroidmanager. org).

5 Gurnell M, Visser TJ, Beck-Peccoz P, Chatterjee VKK: Resistance to thyroid hormone; in Jameson LJ, DeGroot LJ (eds): Endocrinology, Adult and Pediatric, ed 6. Philadelphia, Saunders Elsevier, 2010, vol II, pp 1745-1759.

-6 Jailer JW, Holub DA: Remission of Graves' disease following radiotherapy of a pituitary neoplasm. Am J Med 1960;28:497-499.
7 Atkins D, Best D, Briss PA, Eccles M, FalckYtter Y, Flottorp S, et al: Grading quality of evidence and strength of recommendations. BMJ 2004;328:1490.

8 Guyatt GH, Cook DJ, Jaeschke R, Pauker SG, Schunemann HJ: Grades of recommendation for antithrombotic agents: American College of Chest Physicians Evidence-Based Clinical Practice Guidelines, ed 8. Chest 2008;133(6 suppl):123S-131S. 
-9 Onnestam L, Berinder K, Burman P, Dahlqvist P, Engström BE, Wahlberg J, Nyström HF: National incidence and prevalence of TSHsecreting pituitary adenomas in Sweden. J Clin Endocrinol Metab 2013;98:626-635.

10 Daly AF, Rixhon M, Adam C, Dempegioti A, Tichomirowa MA, Beckers A: High prevalence of pituitary adenomas: a cross-sectional study in the province of Liege, Belgium. J Clin Endocrinol Metab 2006;91:4769-4775.

11 Fernandez A, Karavitaki N, Wass JA: Prevalence of pituitary adenomas: a communitybased, cross-sectional study in Banbury (Oxfordshire, UK). Clin Endocrinol (Oxf) 2010; 72:377-378.

12 Raappana A, Koivukangas J, Ebeling T, Pirilä T: Incidence of pituitary adenomas in Northern Finland in 1992-2007. J Clin Endocrinol Metab 2010;95:4268-4275.

13 Corbetta S, Pizzocaro A, Peracchi M, BeckPeccoz P, Faglia G, Spada A: Multiple endocrine neoplasia type 1 in patients with recognized pituitary tumours of different types. Clin Endocrinol (Oxf) 1997;47:507-512.

14 Daly AF, Tichomirowa MA, Petrossians P, Heliövaara E, Jaffrain-Rea ML, Barlier A, et al: Clinical characteristics and therapeutic responses in patients with germ-line AIP mutations and pituitary adenomas: an international collaborative study. J Clin Endocrinol Metab 2010;95:E373-E383.

$\checkmark 15$ Ma W, Ikeda H, Watabe N, Kanno M, Yoshimoto T: A plurihormonal TSH-producing pituitary tumor of monoclonal origin in a patient with hypothyroidism. Horm Res 2003; 59:257-261.

16 Herman V, Drazin NZ, Gonsky R, Melmed S: Molecular screening of pituitary adenomas for gene mutations and rearrangements. J Clin Endocrinol Metab 1993;77:50-55.

$\checkmark 17$ Asteria C, Anagni M, Persani L, Beck-Peccoz $\mathrm{P}$ : Loss of heterozigosity of the MEN1 gene in a large series of TSH-secreting pituitary adenomas. J Endocrinol Invest 2001;24:796-801.

18 Dong Q, Brucker-Davis F, Weintraub BD, Smallridge RC, Carr FE, Battey J, et al: Screening of candidate oncogenes in human thyro- trope tumors: absence of activating mutations of the Gaq, Ga11, Gas, or thyrotropin-releasing hormone receptor genes. J Clin Endocrinol Metab 1996;81:1134-1140.

19 Gittoes NJ, McCabe CJ, Verhaeg J, Sheppard MC, Franklyn JA: 1998 An abnormality of thyroid hormone receptor expression may explain abnormal thyrotropin production in thyrotropin-secreting pituitary tumors. Thyroid 1998;8:9-14.

20 Ando S, Sarlis NJ, Krishnan J, Feng X, Refetoff $S$, Zhang M, et al: Aberrant alternative splicing of thyroid hormone receptor in a TSHsecreting pituitary tumor is a mechanism for hormone resistance. Mol Endocrinol 2001;15: 1529-1538.

21 Tagami T, Usui T, Shimatsu A, Beniko M, Yamamoto $\mathrm{H}$, Naruse M: Aberrant expression of thyroid hormone receptor beta isoform may cause inappropriate secretion of TSH in a TSH-secreting pituitary adenoma. J Clin Endocrinol Metab 2011;96:E948-E952.

22 Brown RL, Muzzafar T, Wollman R, Weiss RE: A pituitary carcinoma secreting TSH and prolactin: a non-secreting adenoma gone awry. Eur J Endocrinol 2006;154:639-643.

23 Socin HV, Chanson P, Delemer B, et al: The changing spectrum of TSH-secreting pituitary adenomas: diagnosis and management in 43 patients. Eur J Endocrinol 2003;148: 433-442.

24 Bertherat J, Brue T, Enjalbert A, Gunz G, et al: Somatostatin receptors on thyrotropin-secreting pituitary adenomas: comparison with the inhibitory effects of octreotide upon in vivo and in vitro hormonal secretions. J Clin Endocrinol Metab 1992;75:540-546.

25 Brucker-Davis F, Oldfield EH, Skarulis MC, Doppman JL, Weintraub BD: Thyrotropinsecreting pituitary tumors: diagnostic criteria, thyroid hormone sensitivity, and treatment outcome in 25 patients followed at the National Institutes of Health. J Clin Endocrinol Metab 1999;84:476-486.

26 Clarke MJ, Erickson D, Castro MR, Atkinson JL: Thyroid-stimulating hormone pituitary adenomas. J Neurosurg 2008;109:17-22.
27 Sanno N, Teramoto A, Osamura RY: Thyrotropin-secreting pituitary adenomas. Clinical and biological heterogeneity and current treatment. J Neurooncol 2001;54:179-186.

28 Gurnell M, Halsall DJ, Chatterjee VK: What should be done when thyroid function tests do not make sense? Clin Endocrinol (Oxf) 2011;74:673-678.

29 Beck-Peccoz P, Persani L: Thyrotropin-secreting pituitary adenomas; in: Thyroid Disease. www.thyroidmanager.org.

30 Beck-Peccoz P, Persani L: TSH-producing adenomas; in Jameson JL, DeGroot LJ (eds): Endocrinology, ed 5. Philadelphia, Saunders, 2010.

- 31 Beck-Peccoz P, Persani L, Faglia G: Glycoprotein hormone $\alpha$-subunit in pituitary adenomas. Trends Endocrinol Metab 1992;3: 41-45.

32 Beck-Peccoz P, Roncoroni R, Mariotti S, et al: G Sex hormone-binding globulin measurement in patients with inappropriate secretion of thyrotropin (IST): evidence against selective pituitary thyroid hormone resistance in nonneoplastic IST. J Clin Endocrinol Metab 1990;71:19-25.

33 Persani L, Preziati D, Matthews CH, Sartorio A, Chatterjee VK, Beck-Peccoz P: Serum levels of carboxy-terminal cross-linked telopeptide of type I collagen (ICTP) in the differential diagnosis of the syndromes of inappropriate secretion of TSH. Clin Endocrinol (Oxf) 1997;47:207-214.

34 Mannavola D, Persani L, Vannucchi G, et al: Different response to chronic somatostatin analogs in patients with central hyperthyroidism. Clin Endocrinol (Oxf) 2005;62:176181.

35 Losa M, Giovanelli M, Persani L, Mortini P, Faglia G, Beck-Peccoz P: Criteria of cure and follow-up of central hyperthyroidism due to thyrotropin-secreting pituitary adenomas. J Clin Endocrinol Metab 1996;81:30863090.

36 Fliers E, van Furth WR, Bisschop PH: Cure of a thyrotrophin (TSH)-secreting pituitary adenoma by medical therapy. Clin Endocrinol (Oxf) 2012;77:788-790. 